\title{
Botulinum Toxin A in the Treatment of Oropharyngeal or Esophageal Dysphagia
}

\author{
Caroline Beutner $^{1}$, Katharina Bartsch ${ }^{2}$, Harald Schwörer ${ }^{3}$, Rainer Laskawi ${ }^{2}$, Saskia Rohrbach ${ }^{4}$ \\ ${ }^{1}$ Department of Otorhinolaryngology, Duesseldorf University Hospital, Duesseldorf, Germany \\ ${ }^{2}$ Department of Otorhinolaryngology, Georg-August-University Hospital Göttingen, Göttingen, Germany \\ ${ }^{3}$ Department of Gastroenterology and Endocrinology, Georg-August-University Hospital Göttingen, Göttingen, Germany \\ ${ }^{4}$ Department of Audiology and Phoniatrics, Charité, Berlin, Germany \\ Email: Caroline.Beutner@med.uni-duesseldorf.de
}

Received July 22, 2013; revised August 25, 2013; accepted September 10, 2013

Copyright (C 2013 Caroline Beutner et al. This is an open access article distributed under the Creative Commons Attribution License, which permits unrestricted use, distribution, and reproduction in any medium, provided the original work is properly cited.

\begin{abstract}
Objectives: In this retrospective analysis, we explored the effect of botulinum toxin A (BTA) injection to treat oropharyngeal dysphagia or esophageal dysfunction caused by diseases of the upper (UES) and lower (LES) esophageal sphincter. Methods: In total, 48 patients (17 patients with UES disorders, and 31 patients with LES dysfunction; mean age 64 years) were treated between 1996 and 2007 in our hospital. Comorbid diseases as well as maintenance medication were documented to evaluate the overall health status of our patients. The mean duration of symptoms, the number of pre-treatments and the specification of dysphagia were considered. Results: One month after injection, the response rates were 73.3\% (UES group) and 76.6\% (LES group). Most patients in the UES group received 30 - 40 units BTA $\left(\right.$ Botox $\left.^{\circledR}\right)$ whereas most patients in the LES group were treated with 100 units BTA. In cases of re-injection, 50\% of patients in the UES group experienced an escalation of dosage (up to 75 units), whereas the other $50 \%$ received the same dosage. The dosages in the LES group were mostly kept constant. Conclusion: Comparing the two groups with esophageal dysfunction of fundamentally different etiologies (UES/LES), a discrepancy in the level of symptom relief, onset and a longer cessation of clinical benefit were observed in the LES group. In this analysis, we were able to show that injection of BTA is an effective and safe treatment for disorders of the UES and LES.
\end{abstract}

Keywords: Botulinum Toxin; Dysphagia; Upper Esophageal Sphincter; Lower Esophageal Sphincter

\section{Introduction}

Because of the complex course of function during swallowing, the underlying cause of esophageal dysphagia is highly variable. Neuromuscular diseases are especially common. While the treatment of mild disorders of the upper esophageal sphincter (UES) is manageable by swallowing exercises and modification of nutrition, severe disorders in this area often require surgical myotomy of the cricopharyngeal muscle. Disorders of the lower esophageal sphincter (LES), especially achalasia, can be treated with pharmacological agents, such as nitrates, calcium channel blockers, anticholinergic agents and beta-adrenergic agonists, but frequently do not provide satisfactory relief of symptoms [1,2]. Pneumatic dilatation is considered as the treatment of choice, especially in the primary management of achalasia. The standard for surgical management is laparoscopic Heller myotomy, although improvements in long-term remis- sion rates compared to the open approach are still lacking $[1,3]$. Since none of these methods yield universally satisfactory results for either UES or LES diseases, an alternative endoscopic treatment option is proposed. The intramuscular injection of botulinum toxin A (BTA), which is produced by the bacterium Clostridium botulinum, offers a viable alternative treatment. BTA blocks the cholinergic neuromuscular innervation of intra- and extrafusal muscle fibers, innervation of striated and smooth muscle as well as the cholinergic junctions of the autonomous nervous system [4]. Earlier studies reported on the use of BTA in LES- [5,6] and UES [7] -diseases. The purpose of this study is to demonstrate the efficacy of BTA injection as a non-surgical therapeutic option for oropharyngeal or esophageal dysphagia caused by dysfunction of the UES or LES. Furthermore, recommendations for the injection technique and the treatment dosage are stated and any side effects are noted. 


\section{Materials and Methods}

Between 1996 and 2007, 48 patients (female $n=15$, male $n=33$, mean age 64 years, range: 25 - 85 years) with UES or LES dysfunctions were treated at the University Hospital of Goettingen, Germany. Based on their symptoms, medical history and on an interdisciplinary review of the diagnostic results (baseline: flexible laryngoscopy and esophagram in patients presumably having UES dysfunctions; gastrointestinal endoscopy (see Figure 1) and manometry in patients presumably having LES dysfunctions), patients were divided into two groups: UES or LES. MRI, CT-scan or X-ray of the chest were performed in patients with a history of malignant tumors or if malignancy was suspected. For baseline characteristics, see Table 1.

Pre-treatment included pneumatic dilatation, pharmacological or surgical procedures and showed unsatisfactory results (for details see Table 2). Exclusion criteria for the treatment with BTA were pregnancy, nursing, under 18 years of age, malignancies and contraindications for the treatment with BTA or for general anesthesia. A clinical assessment, describing the severity and fre-

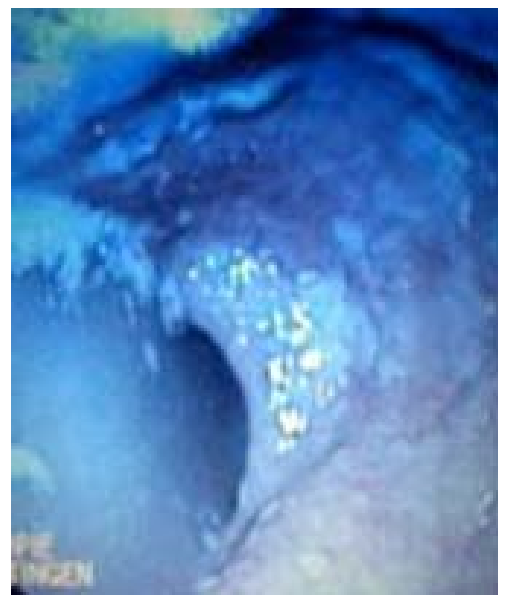

Figure 1. Classical finding from esophago-gastrointestinal endoscopy of a patient suffering from achalasia: prestenotic dilatation and retention of chime.

Table 1. Baseline characteristics of patients with dysphagia caused by disorders of the UES or LES.

\begin{tabular}{cccccccc}
\hline & UES & & & \multicolumn{5}{c}{ LES Total } \\
\hline & & $\begin{array}{c}\text { Neurologic } \\
\text { disease }\end{array}$ & Dystonia & Tumor $\begin{array}{c}\text { Unknown } \\
\text { etiology }\end{array}$ & \\
\hline $\begin{array}{c}\text { Patients } \\
\text { (n) }\end{array}$ & 17 & 7 & 5 & 3 & 2 & 31 & 48 \\
$\begin{array}{c}\text { Age/ } \\
\text { minimum } \\
\text { [years] }\end{array}$ & 25 & 59 & 25 & 42 & 55 & 25 & 25 \\
$\begin{array}{c}\text { Age/ } \\
\text { maximum } \\
\text { [years] }\end{array}$ & 80 & 80 & 75 & 74 & 59 & 85 & 85 \\
\hline
\end{tabular}

quency of the symptoms, was performed 6 months later. For precise localization, the injection into the UES was performed under general anesthesia and direct vision using microscopic controlled endoscopy with a rigid endoscope. After having localized the UES (Figure 2), 30 75 units of botulinum toxin A (BTA, Botox ${ }^{\circledR}$, Allergan Inc., Irvin, CA, USA, freshly reconstituted with $0.9 \%$ sterile saline to a final concentration of $2.5 \mathrm{U} \mathrm{BTA} / 0.1 \mathrm{ml}$ ) were injected into both the lateral, ventral-medial and dorsal-medial part of the cricopharyngeal muscle. An adjusted injection set consisting of a Kleinsasser-Hecht forceps holding a butterfly-needle was used for the injection, as shown in Figure 3.

Table 2. Pre-treatments.

\begin{tabular}{cccc}
\hline Previous therapy & $\begin{array}{c}\text { UES } \\
(n=17)\end{array}$ & $\begin{array}{c}\text { LES } \\
(n=31)\end{array}$ & $\begin{array}{c}\text { Total } \\
(n=48)\end{array}$ \\
\hline Pharmacologic treatment & 10 & 12 & 22 \\
Pneumatic dilatation & 1 & 10 & 11 \\
Percutaneous endoscopic gastrostomy & 10 & 0 & 10 \\
Tracheostomy & 7 & 0 & 7 \\
Logopedics & 8 & 0 & 8 \\
Botulinum toxin injections & 3 & 1 & 1 \\
Surgical myotomy & 0 & 1 & \\
Surgery of the glottis & 1 & 0 & \\
\hline
\end{tabular}

Figure 2. Direct endoscopic exposure of the UES for a safe BTA application into both the lateral, ventral-medial and dorsal-medial part of the cricopharyngeal muscle.

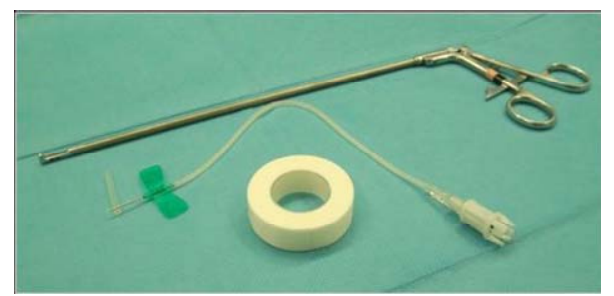

Figure 3. Instruments for transoral BTA injection: adjusted injection set consisting of a Kleinsasser-Hecht forceps holding a butterfly-needle. 
For treatment of the LES, patients were first sedated using midazolam and propofol, placed under cardiovascular monitoring and positioned on their left side; 80 100 units of BTA (Botox ${ }^{\circledR}$ ) were diluted in $0.9 \%$ sterile saline to a final concentration of $2 \mathrm{U}$ BTA/ $0.1 \mathrm{ml}$. After having visualized the sphincteric rosette (Figure 4), 20 units of BTA were injected into each quadrant of the LES and 10 units at each point between quadrants two/ three and four/one (six points) through a $7 \mathrm{~mm}$ sclerotherapy needle. Responders to BTA were defined as patients with symptom relief for at least 2 months. Patients who failed to respond after a single treatment were classified as non-responders without testing for neutralizing antibodies against BTA. These patients were free to elect any alternative treatment options.

To evaluate the overall health status of our patients, we asked them about attendant disorders and any maintenance medications. In addition to the dysphagia, $41.2 \%$ of the UES group had a cerebral infarction or brain hemorrhage; $25.8 \%$ of both groups had a tumor disease and $22.6 \%$ were suffering from reflux esophagitis or gastritis. Maintenance medication in all patients included frequent use of proton pump inhibitors (PPI, 58.5\% in the UES group, $41.9 \%$ in the LES group) and anti-neuromuscular or antispasmodic agents (41.2\% in the UES group); $25.8 \%$ of patients in the LES group used calcium channel blockers, $12.9 \%$ of patients needed prokinetics and $12.0 \%$ used HMG-CoA-reductase enzyme inhibitors.

In patients with severe dysphagia, nutrition was ensured by a permanent endoscopic gastrostomy (PEG). Patients unable to swallow solids were dependent on highcaloric fluid alimentation. In cases of severe achalasia, swallowing liquids was only possible in combination with special maneuvers or positions. In the UES group, besides dysphagia, the primary symptoms were aspiration

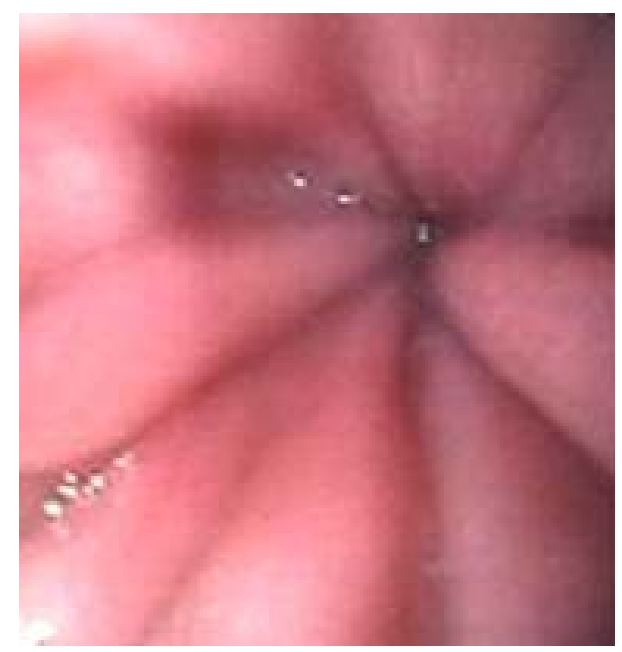

Figure 4. Endoscopic view of the sphincteric rosette of the LES for BTA injections into each quadrant and each point between quadrant two/three and four/one (six points).
( $n=10)$ as shown in Figure 5, dysarthria $(n=8)$ and weight loss $(n=7)$. In the LES group, patients reported a loss of weight $(n=14)$, postprandial vomiting $(n=11)$, regurgitation and retrosternal pain $(n=9)$ in addition to the dysphagia. For duration of symptoms before the treatment see Table 3 .

\section{Results and Analysis}

The 48 patients received a total of 101 injections of BTA into the LES or UES.

In the UES group, ( $n=17)$, the majority (52.9\%) received a single BTA injection, 23.5\% were treated twice, $17.6 \%$ received three injections and one patient received four injections. In those receiving more than one injection the dosage was accelerated in 4 cases and remained unchanged in 4 cases. The initial dosage ranged from 30 to $75 \mathrm{U}$. 29.4\% received $30 \mathrm{U}, 53 \%$ received 40 - $50 \mathrm{U}$, $11.8 \%$ got 51 - $65 \mathrm{U}$ and $5.9 \%$ (one patient) received 75 U. The majority of patients in this group who required a second injection (17.6\%) were treated after an interval of at least 150 days.

In the LES group, $(n=31), 38.7 \%$ received one injec-

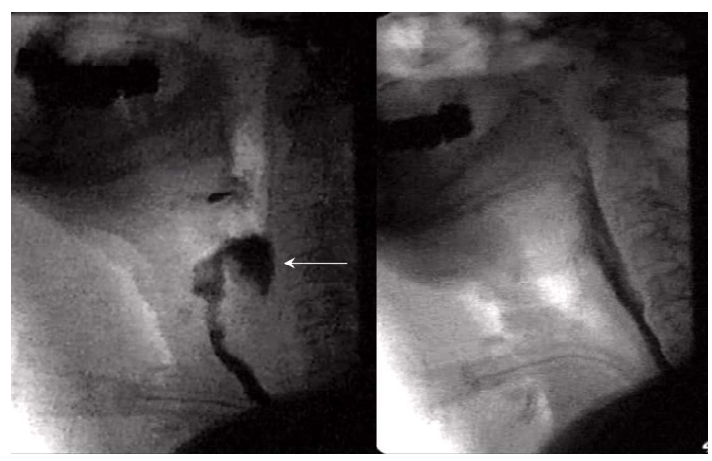

Figure 5. Videofluoroscopy in one representative patient in the UES group, suffering from hypertrophy of the cricopharyngeal muscle before and after treatment with botulinum toxin A. The prestenotic pooling of contrast medium and laryngeal penetration with aspiration is shown in the left image (arrowhead). After injection of BTA, a normalized UES function without retention or aspiration is observed in this patient (right image).

Table 3. Duration of symptoms.

\begin{tabular}{cccc}
\hline Duration of symptoms [months] & $\begin{array}{c}\text { UES } \\
(n=17)\end{array}$ & $\begin{array}{c}\text { LES } \\
(n=31)^{*}\end{array}$ & $\begin{array}{c}\text { Total } \\
(n=48)^{*}\end{array}$ \\
\hline $0-3$ & 5 & 2 & 7 \\
$4-6$ & 4 & 1 & 5 \\
$7-12$ & 0 & 4 & 4 \\
$13-24$ & 2 & 2 & 4 \\
$>24$ & 6 & 21 & 27 \\
Median & 6.5 & $>24$ & $>24$ \\
\hline
\end{tabular}

* One patient without information about the duration of symptoms. 
tion, $19.4 \%$ were treated twice, $22.6 \%$ had three injections, and $35 \%$ received four or more injections. In those receiving more than one injection 16 patients had no change in dosage and in 3 patients the dosage increased. The initial dosage ranged from 40 to $100 \mathrm{U}$. $81 \%$ received $100 \mathrm{U}$. This dosage remained constant in the following four treatments. 3\% were injected with 40 - $50 \mathrm{U}$ and in $16 \%$, due to disconnection of the needle, there was no clear information on the actual dosage. Most patients were retreated after an interval of over 150 days.

In $75.5 \%$ of all patients (combined LES and UES), a good improvement was obtained (responders), while $24.4 \%$ of all patients showed no relief of symptoms after 1 month. In 3 patients, we received no information about the therapeutic effect. After 1 month, the response rate in the LES group was higher (76.6\%) than in the UES group (73.3\%, see Table 4). The effect was noticed after 0 - 2 days in $43.8 \%$ of all patients, while $14.6 \%$ of all patients showed an effect between day 3 and 8 .

Interestingly, in some patients without subjectively reported symptom relief after the treatment, the objective assessment including esophageal manometry, videofluoroscopy and esophagogastroscopy showed an improvement and did not correlate with the persisting complaints of the patients. No severe side effects occurred. In all patients, mild side effects such as reflux ( $n=3)$, pyrosis $(n=3)$, esophagitis/gastritis $(n=1)$ and retrosternal pain $(n=1)$ were manageable by modification of the dosage after the first interval.

\section{Discussion}

The aim of this analysis was to describe the outcome parameters for BTA-based therapy for patients suffering from oropharyngeal or esophageal dysphagia, with regard to the different etiologies of the two entities. Our objective was to evaluate the clinical efficacy, to determine a recommended technique and treatment dosage, and to report on the resultant side effects. The response rates in the UES group (73.3\%) were high compared to an overview of reported studies between 1994 and 2004, [8] describing a success rate varying from $20 \%$ to $100 \%$ [9]. This may be caused by the high initial doses we injected, discussed below, compared to those in the literature.

The number of responders in our LES group (76.6\%) was comparable to those described in the literature. Annese and Bassotti compared 12 of the largest studies (involving at least 30 patients suffering from achalasia) between 1996 and 2004 having response rates of 75\% 93\% after 1 month, [10] of whom 95\% had not been treated before [11]. Even though Storr et al. claim that the same benefit from BTA injections could be expected in untreated or pre-treated patients, [12] a possible explanation for the slightly lower outcome in our group
Table 4. Onset of the effect.

\begin{tabular}{cccc}
\hline Onset of the effect & $\begin{array}{c}\text { UES } \\
(n=17)\end{array}$ & $\begin{array}{c}\text { LES } \\
(n=31)\end{array}$ & $\begin{array}{c}\text { Total } \\
(n=48)\end{array}$ \\
\hline (days) & & & \\
$0-2$ & 4 & 17 & 21 \\
$3-5$ & 1 & 4 & 5 \\
$6-8$ & 2 & 0 & 2 \\
$>8$ & 2 & 0 & 2 \\
Onset unknown & 2 & 2 & 4 \\
No information about the effect & 2 & 1 & 3 \\
No effect & 4 & 7 & 11 \\
\hline
\end{tabular}

could be the high number of pre-treated patients. A lower muscle connective tissue ratio, found in non-responders, [13] could be caused by previous treatments.

The response rates for all patients, irrespective of group, showed no dependence on duration of symptoms before the first treatment. There was a trend towards better outcome in younger patients [14-16] and additional earlier onset of effect in patients with disorders of the LES, especially in patients suffering from severe achalasia, in contrast to patients with UES disorders. This supports the results of a previous study [17].

The number of patients in the LES group, who showed an effect that lasted longer than a minimum of 5 months after the first injection, was higher than in the UES group ( $29 \%$ versus $12 \%$ ). The reason for these variable durations of effect after BTA injections, also observed in disorders of the autonomic nervous system, remains unclear [18]. The discrepancy of symptom relief between the LES group (smooth muscle) compared to the UES group (skeletal muscle) may be caused by the different neurotransmitters at the neuromuscular junctions. A study by Torbey et al. [19] showed a relaxation of smooth muscles in rats in both cholinergic and peptinergic neurons through botulinum toxin injections. In several studies, an earlier onset and a longer cessation of improvement $[5,6,14]$ suggest a higher sensitivity of the smooth muscle to BTA compared to injections in striated muscles. Different predictors of outcome are controversially discussed. Pasricha et al. assessed the decrease in LES pressure as a good predictive factor, [6] whereas others described a correlation of esophageal clearance with symptom improvement [14]. The best predictors of successful outcome after BTA injections into the UES seem to be a good remaining swallowing function, a proven cricopharyngeal muscle hypertonicity [13] or isolated cricopharyngeal muscle pathology [20,21]. Evaluation of outcome is still a challenge because of the discrepancy between symptomatic and objective measures of improvement [3].

The dosages of BTA injected into the UES or LES re- 
ported in the literature are similar to our initial dosages. Dosages from 10 - 100 units (all dosages transformed to Botox $^{\circledR}$ ) with different injections techniques (transoral, percuateneous with or without electromyography, percuataneous CT-guided, with rigid or flexible endoscopes or in open technique during vocal cord lateralization) are reported concerning the UES [7,9,20-24].

We decided to inject dosages of BTA ranging from 30 to $75 \mathrm{U}$ into the UES, with exact localization under general anesthesia, based upon evaluation of the results from dose-range studies. These studies showed a dosage-related effect and relief of symptoms $[25,26]$ without observation of severe complications [8]. Injections of comparable high dosages are usually administered under general anesthesia and direct endoscopic view to ensure safe injection and correct localization of the needle to minimize the risk of complications. The dosages of BTA injection in patients with disorders of the LES, especially of achalasia, vary from $80 \mathrm{U}[6,14]$ to $200 \mathrm{U}[1,11]$. Since the mean dosage used by most centers was about $100 \mathrm{U}$ BTA, in the majority of our patients, we chose to inject this amount. There was no clear dose/effect relationship described in the first injection (investigation of 118 patients suffering from achalasia) [11]. To inhibit the complete nerve regeneration in the smooth muscle, which is responsible for the recurrence of symptoms, a timely re-injection might be useful. Re-injection with 100 U BTA into the LES is recommended after 30 days [11]. Nevertheless, we have to consider that short re-injection intervals and the amount of toxin might risk a receptor blockage by antibodies. The role of antibodies as a result of autoimmune response possibly leading to variable duration of the effect has to be clarified.

The number of complications reported after BTA injection into the UES or LES is low. Uncontrolled diffusion into the hypopharyngeal musculature [22], transient chest pain (also occurring in the placebo group) [14], unilateral vocal fold paresis [9], abnormal acid reflux [27] and aspiration with death after delayed medical intervention [13] have been described. Mediastinitis and ulceration of the esophagus without perforation after BTA injection are rare complications [28]. In our patients, no severe side effects or systemic effects were noted.

The necessity for BTA re-injection, because of its reversible effect, seems disadvantageous, but no additional adverse effects have been described after long-term application [4]. Likewise, the indication for revision in surgery must also be considered. The predictive value of outcome of BTA injection into the UES compared to a myotomy is still controversially discussed $[7,20]$.

While evaluating the different treatment methods available for a patient's relief of dysfunctions of the UES or LES causing dysphagia, numerous factors must be taken into account. These include the endoscopist's or surgeon's experience [13], the risk of complications in surgery, the patient's age, coexisting diseases [29], the overall morbidity of the patient, long-term results and economic factors.

In conclusion, comparing the two groups of esophageal dysfunction with fundamentally different etiologies (UES/LES), intramuscular BTA injections represent a safe and feasible technique with a lack of significant side effects in both groups, especially for older patients with co-morbid illness. Symptom relief, onset and duration of clinical benefit were better in dysfunctions of the LES, explainable by the history of malignant tumor resection in the UES group with scarred tissue and the presumably better effect of BTA in smooth muscles. Further studies are needed providing objective data that correlate with symptom improvement.

\section{Summary}

The pharmacological and surgical treatment of severe oropharyngeal or esophageal dysphagia often gives unsatisfactory results. This paper reports an alternative treatment option, using endoscopic applied intramuscular injection of Botulinum toxin $\mathrm{A}$ in the upper or lower esophageal sphincter. 48 treated patients suffering from oropharyngeal or esophageal dysphagia confirmed the clinical efficacy of this method. The recommended dosage and technique of injection showed no significant side effects.

\section{REFERENCES}

[1] J. E. Leyden, A. C. Moss and P. Mac Mathuna, "Endoscopic Pneumatic Dilatation versus Botulinum Toxin Injection in the Management of Primary Achalasia," Cochrane Database of Systematic Reviews, Vol. 18, No. 4, 2006, Article ID: CD005046. http://dx.doi.org/10.1002/14651858.CD005046.pub2

[2] Z. H. Wen, E. Gardener and Y. P. Wang, "Nitrates for Achalasia," Cochrane Database of Systematic Reviews, 2004, No. 1, Article ID: CD002299. http://dx.doi.org/10.1002/14651858.CD002299.pub2

[3] N. Pehlivanov and P. J. Pasricha, "Achalasia: Botox, Dilatation or Laparoscopic Surgery in 2006," Neurogastroenterology and Motility, Vol. 18, No. 9, 2006, pp. 799804. http://dx.doi.org/10.1111/j.1365-2982.2006.00802.x

[4] D. Dressler, "Pharmacological Aspects of Therapeutic Botulinum Toxin Preparations,” Nervenarzt, Vol. 77, No. 8, 2006, pp. 912-921. http://dx.doi.org/10.1007/s00115-006-2090-2

[5] P. J. Pasricha, W. J. Ravich, T. R. Hendrix, S. Sostre, B. Jones and A. N. Kallo, "Intrasphincteric Botulinum Toxin for the Treatment of Achalasia,” New England Journal of Medicine, Vol. 322, No. 12, 1995, pp. 774-778. http://dx.doi.org/10.1056/NEJM199503233321203

[6] P. J. Pasricha, R. Rai, W. J. Ravich, T. R. Hendrix and A. N. Kalloo, "Botulinum Toxin for Achalasia: Long-Term 
Outcome and Predictors of Response,” Gastroenterology, Vol. 110, No. 5, 1996, pp. 1410-1415. http://dx.doi.org/10.1053/gast.1996.v110.pm8613045

[7] I. Schneider, W. Thumfart and H. E. Eckel, “Treatment of Dysfunction of the Cricopharyngeal Muscle with Botulinum Toxin. Introduction of a New, Non-Invasive Method," Annals of Otology, Rhinology, and Laryngology, Vol. 103, 1994, pp. 31-35.

[8] M. B. J. Moermann and B. J. Mieke, "Cricopharyngeal Botox Injection: Indication and Technique," Current Opinion in Otolaryngology \& Head and Neck Surgery, Vol. 14, No. 6, 2006, pp. 431-436. http://dx.doi.org/10.1097/MOO.0b013e328010b85b

[9] S. I. Atkinson and J. Rees, "Botulinum Toxin for Cricopharyngeal Dysphagia: Case Reports of CT-Guided Injection,” Journal of Otolaryngology, Vol. 26, No. 4, 1997, pp. 273-276.

[10] V. Annese and G. Bassotti, "Non-Surgical Treatment of Esophageal Achalasia,” World Journal of Gastroenterology, Vol. 12, No. 36, 2006, pp. 5763-5766.

[11] V. Annese, G. Bassotti, G. Coccia, M. Dinelli, V. D’Onofrio, G. Gatto, et al. and the GISMAD Achalasia Study Group, "A Multicentre Randomised Study of Intrasphincteric Botulinum Toxin in Patients with Oesophageal Achalasia," Gut, Vol. 46, No. 5, 2000, pp. 597-600. http://dx.doi.org/10.1136/gut.46.5.597

[12] M. Storr, P. Born, E. Frimberger, N. Weigert, T. Rösch, A. Meining, et al., "Treatment of Achalasia: The ShortTerm Response to Botulinum Toxin Injection Seems to be Independent of Any Kind of Pre-Treatment," BMC Gastroenterology, Vol. 2, 2002, p. 19. http://dx.doi.org/10.1186/1471-230X-2-19

[13] G. Zaninotto, R. Marchese-Ragona, C. Briani, M. Costantini, C. Rizzetto, G. Portale, et al., "The Role of Botulinum Toxin Injection and Upper Esophageal Sphincter Myotomy in Treating Oropharyngeal Dysphagia," Journal of Gastrointestinal Surgery, Vol. 8, No. 8, 2004, pp. 997-1006. http://dx.doi.org/10.1016/j.gassur.2004.09.037

[14] C. Cuillere, P. Ducrotté, F. Zerbib, E. H. Metman, D. de Looze, F. Guillemot, et al., "Achalasia: Outcome of Patients Treated with Intrasphincteric Injection of Botulinum Toxin,” Gut, Vol. 41, No. 1, 1997, pp. 87-92. http://dx.doi.org/10.1136/gut.41.1.87

[15] V. M. Fishman, H. P. Parkman, T. D. Schiano, C. Hills, M. A. Dabezies, S. Cohen, et al., "Symptomatic Improvement in Achalasia after Botulinum Toxin Injection of the Lower Esophageal Sphincter," American Journal of Gastroenterology, Vol. 91, No. 9, 1996, pp. 1724-1730.

[16] J. Kolbasnik, E. W. Waterfall, B. Fachnie, Y. Chen and G. Tougas, "Long-Term Efficacy of Botulinum Toxin in Classical Achalasia: A Prospective Study,” American Journal of Gastroenterology, Vol. 94, No. 12, 2004, pp. 34343439. http://dx.doi.org/10.1111/j.1572-0241.1999.01605.x

[17] V. F. Eckardt, C. Aignherr and G. Bernhard, "Predictors of Outcome in Patients with Achalasia Treated by Pneumatic Dilation," Gastroenterology, Vol. 103, 1992, pp. 1732-1738.

[18] M. Naumann, W. H. Jost and K. V. Toyka, "Botulinum
Toxin in the Treatment of Neurological Disorders of the Autonomic Nervous System," Archives of Neurology, Vol. 56, 1999, pp. 914-916. http://dx.doi.org/10.1001/archneur.56.8.914

[19] C. F. Torbey, C. Singaram, P. Bass and E. A. Gaumnitz, "Action of Botulinum Toxin on Gut Smooth Muscle Involves Cholinergic and Non-Cholinergic Neural Pathway," Gastroenterology, Vol. 112, 1997, p. A840.

[20] A. Blitzer and M. F. Brin, "Use of Botulinum Toxin for Diagnosis and Management of Cricopharyngeal Achalasia," Otolaryngology—Head and Neck Surgery, Vol. 116, No. 3, 1997, pp. 328-330. http://dx.doi.org/10.1016/S0194-5998(97)70267-5

[21] G. Y. Shaw and J. P. Searl, "Botulinum Toxin Treatment for Cricopharyngeal Dysfunction,” Dysphagia, Vol. 16, No. 3, 2001, pp. 161-167. http://dx.doi.org/10.1007/s00455-001-0074-8

[22] J. Alberty, M. Oelrich, K. Ludwig, S. Hartmann and W. Stoll, "Efficacy of Botulinum Toxin A for the Treatment of Upper Esophageal Sphincter Dysfunction,” Laryngoscope, Vol. 110, No. 7, 2000, pp. 1151-1156. http://dx.doi.org/10.1097/00005537-200007000-00016

[23] M. Moermann, Y. Callier, C. Dick and H. Vermeersch, "Botulinum Toxin for Dysphagia Due to Cricopharyngeal Dysfunction," European Archives of Oto-Rhino-Laryngology, Vol. 259, No. 1, 2002, pp. 1-3. http://dx.doi.org/10.1007/PL00007520

[24] R. Marchese-Ragona, G. Marioni, D. A. Restivo and A. Staffieri, "Solving Dysphagia Due to Cricopharyngeal Muscle Dysfunction with Botulinum Toxin,” European Archives of Oto-Rhino-Laryngology, Vol. 262, No. 3, 2005, pp. 250-251. http://dx.doi.org/10.1007/s00405-004-0776-2

[25] F. Mancini, G. Sandrini, A. Moglia, G. Nappi and C. Pacchetti, “A Randomized, Double-Blind, Dose-Ranging Study to Evaluate Efficacy and Safety of Three Doses of Botulinum Toxin Type A (Botox) for the Treatment of Spastic Foot,” Neurological Sciences, Vol. 26, No. 1, 2005, pp. 26-31. http://dx.doi.org/10.1007/s10072-005-0378-9

[26] D. Dressler and J. C. Rothwell, "Electromyographic Quantification of the Paralysing Effect of Botulinum Toxin in the Sternomastoid Muscle," European Neurology, Vol. 43, No. 1, 2000, pp. 13-16. http://dx.doi.org/10.1159/000008122

[27] W. E. Waterfall, G. Tougas, B. Fachnie and U. Chauhan, "Clinical and Manometric Response to Botulinum Toxin (Botox) Used as Primary Treatment for Achalasia,” Gastroenterology, Vol. 108, 1995, p. A225.

[28] R. Mac Ever, M. Liptay and Y. Johnson, "A Case of Mediastinitis Following Botulinum Toxin Type A Treatment for Achalasia,” Nature Clinical Practice Gastroenterology \& Hepatology, Vol. 4, No. 10, 2007, pp. 579-582. http://dx.doi.org/10.1038/ncpgasthep0951

[29] L. Dughera, E. Battaglia, D. Maggio, P. Cassolino, P. R. Mioli, A. Morelli, G. Emanuelli, et al., "Botulinum Toxin Treatment of Oesophageal Achalasia in the Old Old and Oldest Old: A 1-Year Follow-Up Study,” Drugs Aging, 2005, Vol. 22, No. 9, pp. 779-783.

http://dx.doi.org/10.2165/00002512-200522090-00006 